\title{
Prognostic gene expression profiling in melanoma: necessary steps to incorporate into clinical practice
}

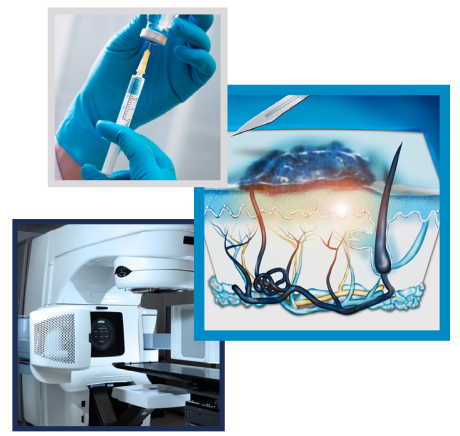

\author{
Douglas Grossman*,1 (D), Caroline C Kim², Rebecca I Hartman ${ }^{3}$, Elizabeth Berry ${ }^{4}$, Kelly C \\ Nelson ${ }^{5}$, Nwanneka Okwundu ${ }^{1}$, Clara Curiel-Lewandrowski ${ }^{6}$, Sancy A Leachman ${ }^{4}$ \& Susan \\ $\mathrm{M} \mathrm{Swetter}^{7}$ \\ ${ }^{1}$ Dermatology, Huntsman Cancer Institute \& University of Utah Health Sciences Center, Salt Lake City, UT 84112, USA \\ ${ }^{2}$ Dermatology, Tufts Medical Center, Boston \& Newton Wellesley Hospital, Wellesley, MA 02111, USA \\ ${ }^{3}$ Dermatology, Brigham \& Women's Hospital, Harvard Medical School, \& Veterans Affairs Boston Healthcare System, Boston, MA \\ 02446, USA \\ ${ }^{4}$ Dermatology, Oregon Health \& Sciences University \& Knight Cancer Institute, Portland, OR 97239, USA \\ ${ }^{5}$ Dermatology, MD Anderson Cancer Center, Houston, TX 77030, USA \\ ${ }^{6}$ Dermatology, University of Arizona Cancer Center, Tucson, AZ 85719, USA \\ ${ }^{7}$ Dermatology, Stanford University Medical Center \& Veterans Affairs Palo Alto Health Care System, Palo Alto, CA 94305, USA \\ *Author for correspondence: doug.grossman@hci.utah.edu
}

\section{Practice points}

\section{Background}

- Gene expression profiling (GEP) tests for cutaneous melanoma (CM) can predict risk of recurrence and metastasis.

- GEP tests are not recommended by national CM care guidelines outside of a clinical study.

- High quality evidence guiding best practice use of GEP tests in CM is lacking.

Prognostic GEP tests for melanoma

- GEP tests measure relative expression of a finite panel of genes using mRNA extracted from tumor tissue slides.

- Available GEP tests for CM include Decision-Dx Melanoma ${ }^{T M}$ and Melagenix.

- Other GEP tests for CM are in clinical development (SkylineDx, Rotterdam, The Netherlands; Natera, CA, USA).

Opportunities \& uncertainties of prognostic GEP testing

- Prognostic GEP testing could improve staging accuracy and help to streamline management.

- Patients identified at increased risk of disease recurrence and metastasis may benefit from enhanced surveillance and newer adjuvant therapies, although neither has demonstrated improved survival in node-negative CM.

- It is not currently known whether a high-risk GEP classification or presymptomatic detection of distant metastasis (through surveillance imaging) is associated with improved response to newer systemic therapies.

Challenges in closing the recommendation gap

- Major challenges of GEP testing are determining which tumor should be tested and how results should affect patient management.

- Additional barriers include resistance to change, reluctance to abandon familiar practices, high costs that may not be borne by insurance and financial disincentives for providers to abandon established procedures.

Potential considerations for clinical trials incorporating prognostic GEP testing

- The goals of future GEP trials would be to establish if GEP testing alone or in conjunction with sentinel lymph node biopsy (SLNB) has greater prognostic value than existing CM staging parameters, and to determine outcomes of different screening and management algorithms based on GEP test results.

- SLNB and GEP testing are not designed to predict the same outcomes, although they may identify overlapping subsets of patients with increased risk for regional and/or distant metastasis.

- Clinical studies are also needed to examine whether adjuvant targeted and immunomodulating agents will benefit GEP-designated high risk, SLNB-negative patients in reducing risk of metastasis and death.

Other considerations

- The cost of a prospective trial assessing impact of GEP testing on clinical outcome would depend on number of subjects enrolled, trial design, positive and negative predictive values of the GEP test employed and the desired magnitude of clinical effect.

- Consideration should be given to use of archived CM specimens collected as part of large cooperative group trials in which patients were randomized to treatment.

- However, it may not be possible to fully assess the value of a GEP test if subjects are not randomized to an intervention based on test results, and it may not be possible to determine superiority of a GEP test compared with other staging tests (like SLNB) if subjects are not randomized to one screening test versus another. 
Prognostic gene expression profiling (GEP) tests for cutaneous melanoma (CM) are not recommended in current guidelines outside of a clinical trial. However, their use is becoming more prevalent and some practitioners are using GEP tests to guide patient management. Thus, there is an urgent need to bridge this gap between test usage and clinical guideline recommendations by obtaining high-quality evidence to guide us toward best practice use of GEP testing in CM patients. We focus here on the opportunities and uncertainties associated with prognostic GEP testing in CM, review how GEP testing was incorporated into clinical care guidelines for uveal melanoma and breast cancer and discuss the role of clinical trials to determine best use in patients with $\mathrm{CM}$.

First draft submitted: 28 September 2019; Accepted for publication: 14 November 2019; Published online: 17 December 2019

Keywords: gene expression profile $\bullet$ melanoma • molecular testing

Molecular prognostic tests based on gene expression profiling (GEP) of cutaneous melanoma (CM) are highly sensitive and have the potential to predict biological behavior. These include the 31-gene GEP Decision-Dx Melanoma $^{T M}$ (Castle Biosciences; TX, USA) and eight-gene GEP Melagenix (NelaCare; Koln, Germany) tests. Other companies with GEP tests for CM in clinical development include: SkylineDx (Rotterdam, The Netherlands) and Natera (CA, USA). We will need high-quality evidence to guide us toward best practice use of these tests for our CM patients. We discuss the opportunities and challenges in implementing prognostic GEP testing in CM, and the role of clinical trials to determine best use for optimal and cost-effective patient care.

Prognostic GEP tests for melanoma

GEP testing involves measuring relative expression of a finite panel of genes using mRNA, extracted from formalinfixed paraffin-embedded tumor tissue slides, that is amplified by reverse transcription PCR. Archival tissue with sufficient tumor remaining to cut multiple slides is required. Prior studies identified patterns of gene expression (i.e., gene signatures) associated with CM tumor progression and metastasis [1-3]. There are several GEP tests for melanoma that are commercially available or in development (Table 1). The 31-GEP test is the best known and most widely used GEP test in the USA. Developed using publicly available gene expression data and an established prognostic test for uveal melanoma (UM) [4], it consists of a panel of 31 genes (28 signature plus 3 control genes). Tumors are classified as either class 1 (low risk) or class 2 (high risk) [5], and both classes are now reported with A or B subclasses to further stratify risk.

Two recent retrospective studies utilizing the 31-GEP test described 690 pooled CM cases (American Joint Committee on Cancer [AJCC] stages I-III) from 18 centers associated with either a metastatic event or $>5$ years of follow-up without metastasis [6,7]. Along with Breslow depth, ulceration and positive sentinel lymph node biopsy (SLNB), a class 2 result was independently associated with metastasis in multivariable analyses. The 31-GEP test further stratified patients with negative SLNB for distant metastatic risk. Similarly, two recent prospective studies (consisting primarily of AJCC stage I/II patients) found GEP class to be the strongest predictor of both local recurrence and distant metastasis $[8,9]$. Finally, in a study of 1421 prospectively tested T1/T2 tumor stage patients - most undergoing SLNB - from 26 centers, GEP class was a significant predictor of SLNB-positivity [10]. The 31-GEP test is now covered by Centers for Medicare and Medicaid Services for patients over age 65 with T1a (with adverse features), $\mathrm{T} 1 \mathrm{~b}$ and $\mathrm{T} 2$ tumors.

The eight-GEP MelaGenix test is based on an eight-gene signature designed to determine risk of relapse of AJCC stage I/II CM patients, and to guide further treatment of stage III CM patients [11]. The MelaGenix test was developed in Germany. A panel of nine of 92 candidate genes assessed in $38 \mathrm{CM}$ tumors was able to discriminate between long- and short-term overall survival. This nine-gene signature comprised six protective genes: keratin 9 (KRT9), dermcidin (DCD), prolactin induced protein (PIP), secretoglobin family 1D member 2 (SCGB1D2), secretoglobin family 2A member 2 (SCGB2A2) and collagen alpha6 (COL6AG), and three risk genes: kelch repeat and BTB (POZ) domain containing 10 (KBTBD10), esophageal cancer-related gene 2 (ECRG2) and hairy and enhancer of split 6 (Drosophila; HESG). Subsequently, differential expression of eight out of the nine genes was also associated with distant metastasis-free survival [12]. 
Table 1. Summary of gene expression profiling tests for cutaneous melanoma.

31-GEP test

- Decision-Dx Melanoma ${ }^{\text {TM }}$ (Castle Biosciences)

- Best known and most widely used GEP test in the USA

- Covered by Centers for Medicare and Medicaid Services for patients over age 65 with T1a (with adverse features), T1b and T2 tumors

- Panel of 31 genes (28 signature plus 3 control genes)

- Results: Class 1 (low risk) and class 2 (high risk)

- Identifies node-negative patients at increased risk for relapse

8-GEP test

- MelaGenix (NelaCare)

- Developed in Germany

- Covered by some private insurances in Germany

- Panel of eight genes (including three control genes)

- Score discriminates between high and intermediate survival risk in Stage II CM

SkylineDx melanoma

- SkylineDx

- Currently in development in collaboration with dermatologists at Mayo Clinic

- Gene panel predicts risk of nodal metastasis in CM patients

Signatera

- Developed by Natera

- Customized 16-gene panel developed from primary tumor

- Circulating tumor DNA (ctDNA) test designed to detect presence of molecular residual disease

- Detection of molecular residual disease up to two years earlier than radiographic imaging in breast, bladder, colorectal, and non-small cell lung cancers, with $98 \%$ positive predictive value for relapse

- Test for CM currently in development

CM: Cutaneous melanoma; GEP: Gene expression profiling.

Another single center study from Germany clinically validated the eight-GEP test in 245 patients with AJCC stage II CM. The MelaGenix score, indicating risk of relapse, was an independent predictor of melanoma-specific survival: the low-score group had 5- and 10-year melanoma-specific survival of $92 \%$, while the high-score group had 5- and 10-year melanoma-specific survival of 82 and 67\%, respectively $(\mathrm{p}=0.018)$ [13]. The eight-GEP test has been available since 2015 and is covered by some private insurances in Germany, however, it has not been approved for use in the USA [11].

SkylineDx, a company primarily focused on developing tests to stratify patients and guide treatment for multiple myeloma, is developing a GEP test for CM in collaboration with dermatologists at Mayo Clinic [14]. The algorithm for the test will be based on the results of a multi-institutional study finding that ITGB3, LAMB1, PLAT and TP53 were associated with nodal metastasis [15]. This multicenter validation cohort study on 309 CM patients showed that aberrant expression of ITGB3 predicts nodal metastasis and may be used to guide decision to perform SLNB [16].

Signatera from Natera is a circulating tumor DNA (ctDNA) test designed to detect presence of molecular residual disease in cancer patients. A panel of 16 tumor-specific clonal variants determined from whole exome sequencing of tumor tissue are measured in peripheral blood. Thus, in contrast to other GEP tests, Signatera directly detects presence of metastatic disease (rather than risk of metastasis) and can be repeated over time to assess CM recurrence or response to therapy. Signatera has demonstrated the ability to detect molecular residual disease up to 2 years earlier than radiographic imaging in breast, bladder, colorectal and non-small-cell lung cancers with a $98 \%$ positive predictive value for relapse [17-21]. In a Phase II trial (INSPIRE) conducted in 70 patients with advanced cancers (including head and neck, triple-negative breast, ovarian cancers and CM), Signatera was used to assess ctDNA at baseline and after the third cycle of treatment with pembrolizumab. There was a strong correlation between reduction in tumor-specific ctDNA and overall survival (adjusted hazard ratio $=0.38 ; \mathrm{p}=0.004$ ), progression-free survival (adjusted hazard ratio $=0.47 ; \mathrm{p}=0.006$ ), and overall clinical response rate [22]. A ctDNA-based test specifically designed for CM is currently in development.

\section{Who is using GEP tests?}

Although current melanoma guidelines from the American Academy of Dermatology [23] and the National Comprehensive Cancer Network (NCCN) [24] recommend further study of GEP tests before incorporation into clinical practice for $\mathrm{CM}$, their use for prognosis is becoming more prevalent. For example, approximately 12,000 31-GEP tests were processed in 2018 [25]. Based on the reported incidence of invasive CM in the USA [26], this may represent up to $5-10 \%$ of invasive CM being subject to GEP testing. 
Table 2. Opportunities, challenges and solutions for prognostic gene expression profiling testing in cutaneous

melanoma.

Opportunities

- Improved prognostication

- Identify patients who may benefit from enhanced imaging and/or adjuvant therapy

- Identify patients who may be spared wider excision, SLNB or adjuvant therapy

Unknowns

- If GEP testing can replace or be used in combination with established staging parameters

- If a GEP test can reliably predict SLNB positivity

- If a GEP test can be used to guide decision to offer adjuvant therapy

- If GEP testing can inform management of stage II and IIIA patients

Challenges

- Determining which patients/tumors should be tested

- How GEP tests should be validated, level of evidence required for guidelines

- Provider resistance to change, reluctance to abandon familiar practices/procedures

- Potential costs that may not be borne by insurance

Solutions

- Prospective rigorous clinical trials; but who would perform them, and cover cost?

- Establish if GEP testing alone or in conjunction with SLNB has greater prognostic value

- Determine clinical outcomes of management algorithms based on GEP test results

CM: Cutaneous melanoma; GEP: Gene expression profiling; SLNB: Sentinel lymph node biopsy.

A recent survey study reported that $20 \%$ of - predominantly US - pigmented lesion experts were routinely using one or more GEP tests for CM and basing clinical management decisions on the results [27]. By contrast, $80 \%$ of respondents had never ordered a GEP test because it was not endorsed in CM guidelines, they did not think it would impact their practice, and/or they felt more validation was necessary [27]. This variability in practice even among experts in the field is concerning and highlights the critical need to elucidate the rationale for use of these tests and to obtain evidence to support incorporation of validated molecular testing into clinical practice.

\section{Opportunities \& uncertainties of prognostic GEP testing}

Prognostic GEP testing has the potential to improve prediction of the biological behavior of melanocytic lesions by objectively defining 'high risk' on a molecular level. Patients identified to be at increased risk of disease recurrence and metastasis may benefit from enhanced surveillance (including imaging and physical examination), as well as newer adjuvant therapies, although neither has yet demonstrated improved survival in node-negative CM [28]. While conventional staging parameters such as Breslow depth and SLNB status are strongly correlated with clinical outcomes [29], they may under- or over-estimate metastatic risk. For example, $2 \%$ of patients with thin (T1a) tumors will ultimately experience relapse and death from melanoma [29], and 5\% of all patients with a negative SLNB will develop local recurrence or distant metastasis [30]. On the other hand, there are patients with thick primary tumors or positive SLNB who do not relapse and instead demonstrate long-term survival without additional therapy.

Validated GEP testing could improve staging accuracy and help streamline management (Table 2). For instance, GEP test results could inform which patients might benefit from more, or less aggressive treatment, such as wider or narrower excision margins for their primary CM, undergoing or avoiding a SLNB procedure and receiving or foregoing surveillance imaging or systemic adjuvant therapy. However, it is not currently known whether a high-risk GEP classification or presymptomatic detection of distant metastasis (through surveillance imaging) is associated with improved response to newer systemic therapies. Moreover, if a node-negative patient has a GEP test indicating high risk, it is unlikely that insurance payors would approve expensive adjuvant therapy that is not currently US FDA-approved for node-negative patients.

\section{Challenges in closing the recommendation gap}

Since GEP testing is not currently endorsed in CM practice guidelines outside of a clinical trial or study [23,24], what evidence is needed for its adoption as standard of care? Such guidelines are established by rotating panels of interdisciplinary experts and patient advocates based on review of worldwide evidence. Without a strong scientific basis, there are potential hazards in deviating from clinical practice guidelines if the intervention lacks benefit or causes harm to the patient, as well as potential liability to the provider if there is an unfavorable outcome. At the same time, we have an obligation to proactively advance promising new technologies that may make a difference for our patients in the future. 
The major challenges of GEP testing are determining which tumor should be tested and how GEP tests should be validated (Table 2). It is also important to note additional barriers to adopting any new technology, which includes resistance to change, reluctance to abandon familiar practices, high costs that may not be borne by insurance and financial disincentives for providers to abandon established procedures.

\section{Paradigms for incorporating prognostic GEP testing into practice guidelines}

What approach would best inform practice guideline development for GEP testing for CM (Table 2)? Should CM specialists conduct a multicenter prospective trial? Should this be done from multiple institutions with single or different study designs? Should trials be coordinated by CM/cooperative oncology groups? Who would pay for the trials? Or should various GEP tests simply be disseminated without specific use recommendations, with the hope that best practices will become evident over time? To evaluate these questions, it is instructive to review how GEP testing was incorporated into clinical guidelines for UM and breast cancer.

The NCCN guidelines for UM recommend GEP testing (i.e., DecisionDx-UM ${ }^{\top M}$ ) along with other molecular tests (chromosomal and targeted mutational analysis) if tumor tissue is available [31]. This recommendation was based upon a single prospective 12-center cooperative group study in 459 UM patients demonstrating that over $97 \%$ of cases were correctly classified, and that GEP test results had superior independent prognostic value compared to other existing prognostic factors such as chromosome 3 status [32]. A subsequent study reported that UM tumors with low-risk GEP test results respond more rapidly to plaque radiotherapy [33]. There was a consensus among practitioners that GEP testing, used in conjunction with other molecular tests, would be useful to help identify patients who may benefit from certain clinical trials and enhanced surveillance [34], although systemic therapies have not yet altered the outcome of metastatic UM. The NCCN recommends imaging intervals for UM patients with high-risk GEP test results every 3-6 months (vs every 6-12 months for low-risk GEP test results) [31].

A second prospective, multicenter study of 70 UM patients validated the prognostic value of GEP testing and reported that those with high-risk GEP test results received significantly higher intensity monitoring and higher oncology/clinical trial enrollment compared with those with low-risk test results [35]. However, there do not yet appear to be any published studies demonstrating that enhanced surveillance based on GEP testing improves clinical outcomes in UM. Why do some practitioners [27] feel that GEP testing in CM should be subjected to more rigorous validation linked to clinical outcomes than was applied in UM? There are significant differences between CM and UM, namely that UM is much less common than CM and treated by a relatively small number of specialized practitioners, staging in UM is based on tumor size and presence of distant metastasis, and there is no alternative staging procedure for UM (e.g., SLNB). The introduction of GEP testing in UM may have been less controversial because it did not disrupt current clinical practice, represented an additional molecular test to those already routinely performed, and did not threaten to displace established and well-validated staging procedures (e.g., SLNB). A high-risk GEP result in UM leads only to increased imaging surveillance but not systemic therapy, thus the possible downstream costs of GEP testing in CM are potentially significantly higher. Finally, unlike $\mathrm{UM}$, patients with CM who recur often have outstanding responses to either immunotherapy or BRAF-directed therapies. For these reasons, some may feel that GEP testing in CM should be subjected to more rigorous validation linked to clinical outcomes than was applied in UM.

Oncotype Dx ${ }^{\circledR}$ (Genomic Health, CA, USA) is currently endorsed by NCCN as a prognostic 21-gene GEP test for breast cancer [36]. After the publication and review of multiple prospective validation studies in $>1300$ patients [37,38], Oncotype Dx was first incorporated in clinical guidelines in 2007 in the American Society of Clinical Oncology recommendations to identify patients with node-negative, estrogen receptor (ER)-positive breast cancer who would derive the most benefit from adjuvant tamoxifen (endocrine therapy) and who may not require adjuvant chemotherapy [39]. In 2008, Oncotype Dx was added to NCCN recommendations to stratify recurrence risk and possible benefits of adjuvant chemotherapy for women with node-negative, ER-positive, HER2-negative breast cancer [40]. Following publication of the prospective Trial Assigning Individualized Options for Treatment (TAILORx) in which 10,273 women with moderate Oncotype Dx recurrence risk demonstrated similar disease free survival when randomized to receive either endocrine or chemo-endocrine adjuvant therapy [41], NCCN guidelines were updated in 2018 recommending Oncotype Dx as the preferred test to determine which patient may not need chemotherapy [36]. For patients with micrometastases and positive nodes, the NCCN guidelines recommend considering one of several commercially available prognostic GEP tests to determine chemotherapy benefit [36]. 


\section{Potential considerations for clinical trials assessing benefit of prognostic GEP testing}

Do we need prospective, randomized clinical trials to provide required data to inform practice guidelines for GEP testing, similar to those performed for wide excision $[42,43]$ and SLNB [44,45] for primary CM? The goals of future GEP trials would be twofold: to establish if GEP testing alone or in conjunction with SLNB has greater prognostic value than existing $\mathrm{CM}$ staging parameters; and to determine outcomes of different screening and management algorithms based on GEP test results. There are two basic paradigms for randomized trials incorporating GEP testing in CM. First, patients can be randomized to treatment based on GEP test results, as was done in the breast cancer and UM trials noted above. Alternatively, patients can be randomized to staging test (e.g., SLNB or GEP testing). However, it is important to recognize that SLNB and GEP testing are not designed to predict the same outcomes, although they may identify overlapping subsets of patients with increased risk for regional and/or distant metastasis. Notably, lymphatic (better predicted by SLNB) and hematogenous (better predicted by GEP tests) dissemination of early-stage CM is associated with overlapping but distinct risk factors [46].

It is important to note that guideline-based recommendations of GEP testing in breast cancer followed testing its prognostic ability in node negative, ER-positive patients, which resulted in recommendations specific to that cancer cohort. A similar structured approach is needed for GEP testing in CM to identify the subset (i.e., specific sub-stages) of patients who are most likely to benefit, as pretest probability will likely vary depending on tumor stage for any test. While trials should be based on the principle of equipoise, and not direct patients to a substandard course, there are potential inherent hazards associated with any approach. For example, patients could be identified who would not otherwise be offered additional surveillance or treatment per CM guidelines but (if GEP test indicates high risk) could benefit from a intervention. However, this must be weighed against the cost of the imaging, harms of more invasive testing from false-positive imaging results, potential adverse side effects of systemic therapy and increased patient anxiety. On the other hand, patients who would otherwise be offered imaging or adjuvant systemic therapy may be spared the cost and risk of those interventions in the setting of a low-risk GEP classification, although incorrect risk classification may cause harm if potentially beneficial interventions are not offered. In addition, studies are needed to examine whether adjuvant targeted and immunomodulating agents will benefit GEP-designated high risk, SLNB-negative patients in reducing risk of metastasis and death.

\section{Other considerations}

The cost of a prospective trial assessing impact of GEP testing on clinical outcomes would depend on number of subjects enrolled, trial design, positive and negative predictive values of the GEP test employed and the desired magnitude of clinical effect. An additional consideration is the pretest probability of a GEP test in specific groups of patients. For example, an analysis of outcomes for the 31-GEP test in patients with T1 tumors (where sensitivity and positive predictive values were $10-20 \%$ and specificity and negative predictive values were $90-96 \%$ ) estimated that up to $90 \%$ of patients who tested positive would be predicted not to develop metastasis ( $90 \%$ false-positive rate) while $80 \%$ of patients who ultimately developed metastasis would be predicted to test negative ( $80 \%$ false-negative rate) [47]. It is likely that the performance of most GEP tests will be better in higher compared with lower stage patients. It is also important to note that despite the availability of the multiple GEP testing platforms discussed above, it is likely that for most CM primary tumors there will only be enough remaining tissue in the paraffin block to accommodate a single GEP test.

Finally, consideration should also be given to use of archived CM specimens collected as part of large cooperative group trials in which patients were randomized to treatment (such as adjuvant therapies). GEP test results could be correlated with clinical course and treatment outcomes. This approach would be far cheaper, easier to implement and avoids any additional risks for the patient. However, it may not be possible to fully assess the value of a test if subjects are not randomized to an intervention based on test results. In addition, it may not be possible to determine superiority of a GEP test compared with other staging tests (like SLNB) if subjects are not randomized to one screening test versus another. Thus, prospective randomized trials may subsequently be needed.

\section{Future perspective}

We expect that prognostication in CM will continue to evolve by incorporating molecular features of the tumor with traditional clinic-pathologic features in order to best estimate individual risk. Although GEP testing in CM is promising, it is currently being used by some practitioners to influence management without endorsement of national guidelines. It will be difficult to determine over time whether a certain GEP test benefits patients if results are not incorporated into a clinical study examining patient outcomes. Currently, it is not clear which GEP test 
is superior among several platforms, and which test(s) is most appropriate for patients at each tumor stage. While some GEP tests are currently commercially available without clinical studies demonstrating which tumors/patients benefit from testing, other GEP tests are being developed and tested prior to commercial release. Ideally, the performance (in terms of sensitivity, specificity, positive and negative predictive value) of a GEP test should justify its application in identifying which patients can be spared unneeded interventions and which may benefit from additional interventions that would not otherwise be recommended. We believe that carefully designed clinical trials will guide us toward the best practice and clinical use of these tests for CM patients. We look forward to collaborative efforts among dermatologists, medical oncologists and surgical oncologist to perform these studies. With high-quality data to inform which GEP tests will benefit which CM patients with specific sub-stages and clinical settings, broader use of GEP is expected and would be appropriate in the context of high-quality clinical validation studies.

\section{Acknowledgments}

We appreciate helpful discussions with J Vetto, K Grossmann, G Bowen, J Hyngstrom, E Buchbinder, A Ribas and J Kirkwood during the preparation of this manuscript.

\section{Financial \& competing interests disclosure}

D Grossman received honoraria for serving on the advisory board of Orlucent, Inc.; E Berry received honoraria for serving on the advisory board of Bristol-Myers Squibb; C Curiel-Lewandrowski received honoraria for serving as a consultant for Bristol-Myers Squibb and an investigator grant from Amgen; SA Leachman received honoraria for serving on the advisory boards of Orlucent, Inc and Merck.

The authors have no other relevant affiliations or financial involvement with any organization or entity with a financial interest in or financial conflict with the subject matter or materials discussed in the manuscript apart from those disclosed.

No writing assistance was utilized in the production of this manuscript.

\section{Open access}

This work is licensed under the Attribution-NonCommercial-NoDerivatives 4.0 Unported License. To view a copy of this license, visit http://creativecommons.org/licenses/by-nc-nd/4.0/

\section{References}

Papers of special note have been highlighted as: $\bullet$ of interest

1. Smith AP, Hoek K, Becker D et al. Whole-genome expression profiling of the melanoma progression pathway reveals marked molecular differences between nevi/melanoma in situ and advanced-stage melanomas. Cancer Biol. Ther. 4(9), 1018-1029 (2005).

2. Haqq C, Nosrati M, Sudilovsky D et al. The gene expression signatures of melanoma progression. Proc. Natl Acad. Sci. USA 102(17), 6092-6097 (2005).

3. Jaeger J, Koczan D, Thiesen HJ et al. Gene expression signatures for tumor progression, tumor subtype, and tumor thickness in laser-microdissected melanoma tissues. Clin. Cancer Res. 13(3), 806-815 (2007).

4. Onken MD, Worley LA, Char DH et al. Collaborative ocular oncology group report number 1: prospective validation of a multi-gene prognostic assay in uveal melanoma. Ophthalmology 119(8), 1596-1603 (2012).

5. Gerami P, Cook RW, Wilkinson J et al. Development of a prognostic genetic signature to predict the metastatic risk associated with cutaneous melanoma. Clin. Cancer Res. 21(1), 175-183 (2015).

6. Zager JS, Gastman BR, Leachman S et al. Performance of a prognostic 31-gene expression profile in an independent cohort of 523 cutaneous melanoma patients. BMC Cancer. 18(1), 130 (2018).

7. Gastman BR, Gerami P, Kurley SJ et al. Identification of patients at risk of metastasis using a prognostic 31-gene expression profile in subpopulations of melanoma patients with favorable outcomes by standard criteria. J. Am. Acad. Dermatol. 80(1), 149-157 (2019).

- Use of the 31-gene expression profiling (GEP) test to identify a subset of patients with negative sentinel lymph node biopsy and increased risk of metastasis.

8. Keller J, Schwartz TL, Lizalek JM et al. Prospective validation of the prognostic 31-gene expression profiling test in primary cutaneous melanoma. Cancer Med. 8(5), 2205-2212 (2019).

9. Podlipnik S, Carrera C, Boada A et al. Early outcome of a 31-gene expression profile test in 86 AJCC stage IB-II melanoma patients. A prospective multicentre cohort study. J. Eur. Acad. Dermatol. Venereol. 33(5), 857-862 (2019).

10. Vetto JT, Hsueh EC, Gastman BR et al. Guidance of sentinel lymph node biopsy decisions in patients with T1-T2 melanoma using gene expression profiling. Future Oncol. 15(11), 1207-1217 (2019). 
11. MelaGenix-The New Multi-Gene Expression Test (2019). https://www.melagenix.com/medizinische-fachkreise.html

12. Brunner GM, Reitz A, Heinecke A et al. A nine-gene signature predicting clinical outcome in cutaneous melanoma. J. Cancer Res. Clin. Oncol. 139(2), 249-258 (2013).

- Use of the Melagenix test to discriminate patients with different distant metastasis-free survival.

13. Garbe C, Sinnberg T, Niessner H, Sülberg H, Eigentler TK, Amaral T. Clinical validation of a prognostic 11-gene assay in prospectively collected FFPE tissue of patients with AJCC v8 stage II cutaneous melanoma (CM). Presented at: The American Society of Clinical Oncology. IL, USA, 31 May-3 June (2019).

14. $360 \mathrm{dx}$ Skyline Dx, Mayo Clinic to Develop Diagnostic Tests for Melanoma (2019). https://www.360dx.com/business-news/skylinedx-mayo-clinic-develop-diagnostic-tests-melanoma\#.XWQWl-NKhEY

15. Meves A, Nikolova E, Heim JB et al. Tumor cell adhesion as a risk factor for sentinel lymph node metastasis in primary cutaneous melanoma. J. Clin. Oncol. 33(23), 2509-2515 (2015).

- Description of prognostic markers used for the Skyline Dx Melanoma test.

16. Meves A, Weaver AL, Saunte DM et al. avß3 Integrin-linked 'stromal response' expression signature to identify high-risk melanoma: report on a 309 patient multi-center validation cohort. Presented at: American Academy of Dermatology Annual Meeting. CA, USA, 16-20 February (2018).

17. Sethi H, Salari R, Navarro $S$ et al. Analytical validation of the Signatera ${ }^{T M}$ RUO assay, a highly sensitive patient-specific multiplex PCR NGS-based noninvasive cancer recurrence detection and therapy monitoring assay. Presented at: American Association for Cancer Research Annual Meeting. IL, USA, 14-18 April (2018).

18. Reinert T, Henriksen TV, Christensen E et al. Analysis of plasma cell-free DNA by ultradeep sequencing in patients with stages I to III colorectal cancer. JAMA Oncol. (2019) doi:10.1001/jamaoncol.2019.0528 (Epub ahead of print).

19. Christensen E, Birkenkamp-Demtröder K, Sethi H et al. Early detection of metastatic relapse and monitoring of therapeutic efficacy by ultra-deep sequencing of plasma cell-free DNA in patients with urothelial bladder carcinoma. J. Clin. Oncol. (2019) doi: 10.1200/JCO.18.02052 (Epub ahead of print).

20. Abbosh C, Birkbak NJ, Wilson GA et al. Phylogenetic ctDNA analysis depicts early-stage lung cancer evolution. Nature 545(7655), 446-451 (2017).

21. Coombes RC, Page K, Salari R et al. Personalized detection of circulating tumor DNA antedates breast cancer metastatic recurrence. Clin. Cancer Res. 25(14), 4255-4263 (2019).

- Use of Signatera circulating tumor DNA test to predict recurrence in breast cancer.

22. Iafolla MAJ, Yang C, Dashner $S$ et al. Bespoke circulating tumor DNA (ctDNA) analysis as a predictive biomarker in solid tumor patients treated with single agent pembrolizumab. Presented at: The American Society of Clinical Oncology.IL, USA, 31 May-1 June (2019).

23. Swetter SM, Tsao H, Bichakjian CK et al. Guidelines of care for the management of primary cutaneous melanoma. J. Am. Acad. Dermatol. 80(1), 208-250 (2019).

24. Coit DG, Thompson JA, Albertini MR et al. Cutaneous melanoma, version 2.2019, NCCN clinical practice guidelines in oncology. J. Natl Compr. Canc. Netw. 17(4), 367-402 (2019).

25. Castle Biosciences.. Castle biosciences announces medicare coverage for the DecisionDx-Melanoma test in cutaneous melanoma. Press release: https://ir.castlebiosciences.com/news-releases/news-release-details/castle-biosciences-announces-medicare-coverage-decisiondx

26. National Cancer Institute: Cancer Stat Facts: Melanoma of the Skin (2019). https://seer.cancer.gov/statfacts/html/melan.html

27. Varedi A, Gardner LJ, Kim CC, Chu EY, Ming ME, Curiel-Lewandrowski C et al. Use of new molecular tests for melanoma by pigmented lesion experts. J. Am. Acad. Dermatol. (2019) doi: 10.1016/j.jaad.2019.08.022 (Epub ahead of print).

\section{- Highlights gap in GEP test usage patterns and national guideline recommendations.}

28. Klapperich ME, Bowen GM, Grossman D. Current controversies in early-stage melanoma: questions on management and surveillance. J. Am. Acad. Dermatol. 80(1), 15-25 (2019).

29. Gershenwald JE, Scolyer RA, Hess KR et al. Melanoma staging: evidence-based changes in the American Joint Committee on Cancer eighth edition cancer staging manual. CA Cancer J. Clin. 67(6), 472-492 (2017).

30. Valsecchi ME, Silbermins D, de Rosa N, Wong SL, Lyman GH. Lymphatic mapping and sentinel lymph node biopsy in patients with melanoma: a meta-analysis. J. Clin. Oncol. 29(11), 1479-1487 (2011).

31. Barker CA, Salama AK. New NCCN guidelines for uveal melanoma and treatment of recurrent or progressive distant metastatic melanoma. J. Natl Compr. Canc. Netw. 16(5S), 646-650 (2018).

32. Onken MD, Worley LA, Char DH et al. Collaborative ocular oncology group report number 1: prospective validation of a multi-gene prognostic assay in uveal melanoma. Ophthalmology 119(8), 1596-1603 (2012).

33. Mruthyunjaya P, Seider MI, Stinnett S, Schefler A. Association between tumor regression rate and gene expression profile after iodine 125 plaque radiotherapy for uveal melanoma. Ophthalmology 124(10), 1532-1539 (2017).

34. Aaberg TM, Cook RW, Oelschlager K, Maetzold D, Rao PK, Mason JO. Current clinical practice: differential management of uveal melanoma in the era of molecular tumor analyses. Clin. Ophthalmol. 8, 2449-2460 (2014). 
35. Plasseraud KM, Cook RW, Tsai T et al. Clinical performance and management outcomes with the DecisionDx-UM gene expression profile test in a prospective multicenter study. J. Oncol. 2016, 5325762 (2016).

36. Goetz MP, Gradishar WJ, Anderson BO et al. NCCN Guidelines Insights: Breast Cancer, Version 3.2018. J. Natl Compr. Canc. Netw. 17(2), 118-126 (2019).

37. Paik S, Shak S, Tang G et al. A multigene assay to predict recurrence of tamoxifen-treated, node-negative breast cancer. $N$. Engl. J. Med. 351(27), 2817-2826 (2004).

38. Paik S, Tang G, Shak $S$ et al. Gene expression and benefit of chemotherapy in women with node-negative, estrogen receptor-positive breast cancer. J. Clin. Oncol. 24(23), 3726-3734 (2006).

39. Harris L, Fritsche H, Mennel R et al. American Society of Clinical Oncology update of recommendations for the use of tumor markers in breast cancer. J. Clin. Oncol. 25(33), 5287-5312 (2007).

40. Carlson RW, Allred DC, Anderson BO et al. Breast cancer. Clinical practice guidelines in oncology. J. Natl Compr. Canc. Netw. 7(2), 122-192 (2009).

41. Sparano JA, Gray RJ, Makower DF et al. Adjuvant chemotherapy guided by a 21-Gene expression assay in breast cancer. N. Engl. J. Med. 379(2), 111-121 (2018).

42. Balch CM, Urist MM, Karakousis CP et al. Efficacy of 2-cm surgical margins for intermediate-thickness melanomas (1 to $4 \mathrm{~mm}$ ). Results of a multi-institutional randomized surgical trial. Ann. Surg. 218(3), 262-267 (1993).

43. Veronesi U, Cascinelli N, Adamus J et al. Thin stage I primary cutaneous malignant melanoma. Comparison of excision with margins of 1 or 3 cm. N. Engl. J. Med. 318(18), 1159-1162 (1988).

44. Morton DL, Thompson JF, Essner R et al. Validation of the accuracy of intraoperative lymphatic mapping and sentinel lymphadenectomy for early-stage melanoma: a multicenter trial. Multicenter Selective Lymphadenectomy Trial Group. Ann. Surg. 230(4), 453-463 (1999).

45. Faries MB, Thompson JF, Cochran AJ et al. Completion dissection or observation for sentinel-node metastasis in melanoma. $N$. Engl. J. 376(23), 2211-2222 (2017).

46. Calomarde-Rees L, García-Calatayud R, Requena Caballero C et al. Risk factors for lymphatic and hematogenous dissemination in patients with stages I to II cutaneous melanoma. JAMA Dermatol. 155(6), 679-687 (2019).

47. Marchetti MA, Bartlett EK, Dusza SW, Bichakjian CK. Use of a prognostic gene expression profile test for T1 cutaneous melanoma: will it help or harm patients? J. Am. Acad. Dermatol. 80(6), e161-e162 (2019).

- Illustrates potential hazards of GEP test usage in early-stage cutaneous melanoma patients. 